\title{
Logistics Distribution System Based on Internet of Things
}

\author{
Xiaohan Li and Jialiang He \\ College of Information and Communication Engineering, Dalian Nationalities University, China
}

\begin{abstract}
This product that can provide distribution services and improve the utilization rate of freight resources. Its construction goal is to use high-tech means to achieve intelligent logistics as the core, greatly accelerating the circulation speed of the goods in the logistics process, reducing manual operation errors, reducing the cost of management, realizing resource utilization and work automation, optimization, simplification to maximize.
\end{abstract}

\section{Keywords-internet of things; logistics distribution}

\section{INTRODUCTION}

IoT has been used in many domains of taffic and logistics[1-5]. Currently domestic and international logistics distribution system although most across a simple delivery of phase, but on level it is still in the traditional sense of the logistics distribution, also many links of logistics and distribution caused huge cost, manpower, and a waste of time. With the rapid development of electronic technology, computer hardware and software technology and enterprise's own development needs, logistics enterprises must attach importance to the logistics distribution system of information management to reduce the cost of logistics. Therefore, it is of great significance to study the intelligent distribution system of the Internet of things. This paper proposes a solution to realize the logistics distribution based on the Internet of things.

\section{SYSTEM INTRODUCTION}

This project uses the high performance cross platform development language Java and the multi user, the multi thread database related knowledge, realizes the user demand data structure, the independence and the sharing. The overall design includes the function of the design system, the overall function framework of the system realization, the realization scheme of the selection function, the reasonable design of the database structure and so on.

First, according to the results of analysis system, the complex is divided into simple sub function modules. The following is the main functional diagram of the logistics distribution system, as shown in Table I.

On the basis of the system function design, doing database design, database design optimization conceptual structure, logical model and physical storage structure, the data in the system are in some form of organization, to realize the data storage, query, editing, searching and so on. On the basis to meet the needs of users, set aside a certain space for development, is convenient for the user to increase in the future new demand, and can effectively store data, improve the efficiency of the system, and guarantee the system of relative stability.

TABLE I. SYSTEM ADMINISTRATOR AND LOGISTICS

\begin{tabular}{|c|c|}
\hline Functional module & Realization function \\
\hline \multirow{5}{*}{ Home page } & Navigation \\
\hline & Advertising position \\
\hline & Supply dynamics \\
\hline & Vehicle source dynamic \\
\hline & News information \\
\hline \multirow{3}{*}{ Login } & Login \\
\hline & Register \\
\hline & Exit \\
\hline Release supply & Release supply \\
\hline Look for foods & Look for foods \\
\hline Look for cars & Look for cars \\
\hline \multirow{2}{*}{ Message } & Waybill message \\
\hline & System message \\
\hline App WebService & App WebService \\
\hline Functional module & Realization function \\
\hline \multirow{11}{*}{ Personal Center } & Common vehicle \\
\hline & Own vehicle \\
\hline & Released resources \\
\hline & Waybill management \\
\hline & Capital account \\
\hline & My points \\
\hline & My discount coupons \\
\hline & Account security \\
\hline & User data \\
\hline & Real name authentication \\
\hline & Call record \\
\hline
\end{tabular}

The web system is the core of the whole logistics picking system, in addition to the terminal app have the function, WebService interface also includes in the web system and its detailed function module is shown in the following table, as shown in Figure I. 


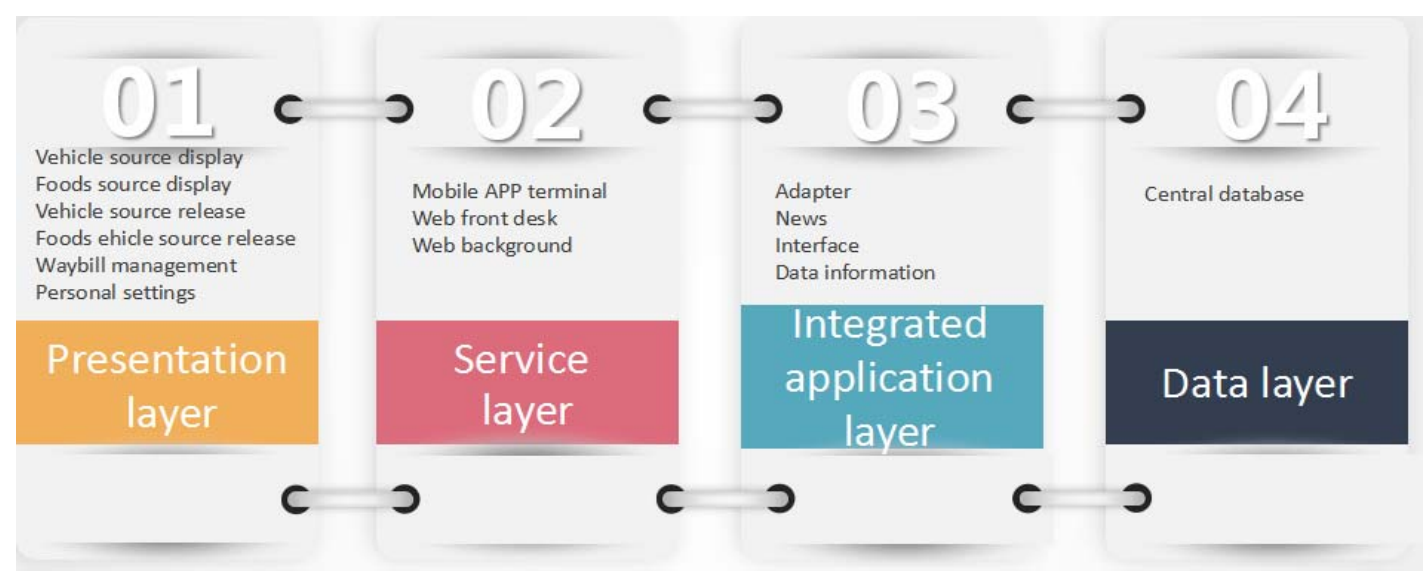

FIGURE I. SYSTEM FUNCTIONAL BLOCK DIAGRAM

Database concept structure design, will looking for the car, looking for goods, personal Center for navigation, the user is indexed, as shown in Figure II.
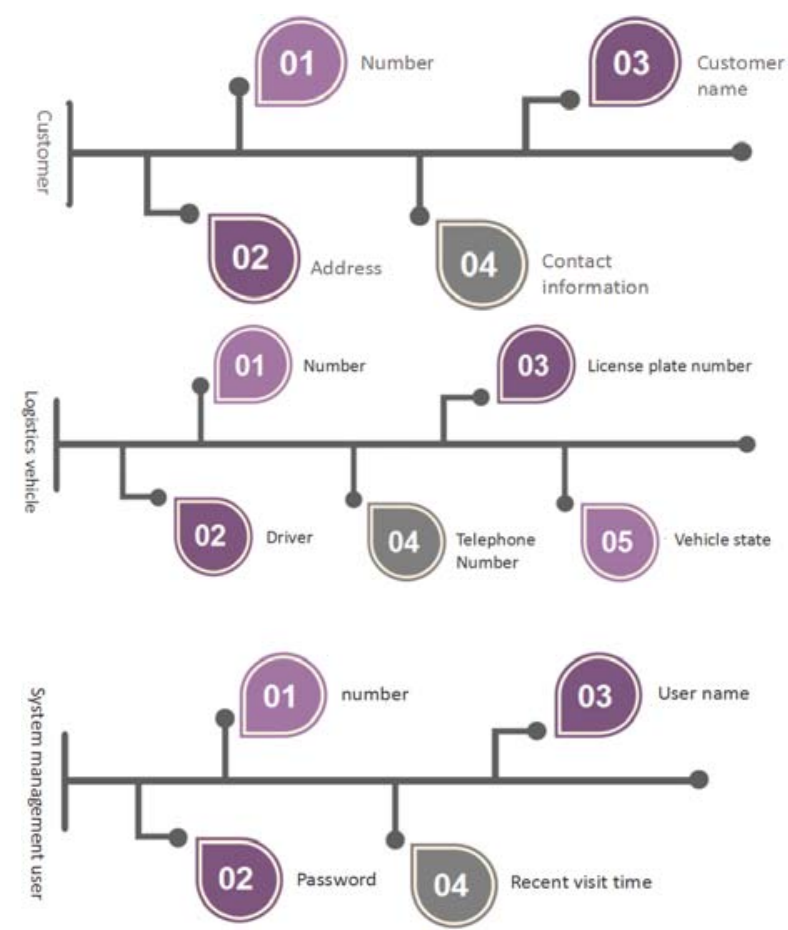

FIGURE II. DESIGN OF DATABASE CONCEPTUAL STRUCTURE

After the concept of database structure design, carry out the logic structure design of database, turning the conceptual model into a logical model, which is the e-r diagram of the entities, relationships, attributes into a database supported by the data structure of process, to achieve entity integrity and referential integrity and user-defined integrity. Involving the main business of design data table: the administrator information table, the customer information table, logistics vehicles information table, invoice information table, etc.

The logistics distribution system run the simulation , for suppliers search supply of goods which conform to the owner transport line, for truck drivers search options which are in accordance with the shipper delivery destination, as shown in Figure III.

Taking Dalian City as a model, Dalian city is divided into multiple zones, each zone has main road. When the system is running, position is provided for supplier and truck drivers as a criteria, let the spaced closer supplier and truck drivers random matching, and open the map mode, make the whole matching and the receiving process more efficient, more convenient. As shown in Figure IV.

After the concept of database structure design, carry out the logic structure design of database , turning the conceptual model into a logical model ,which is the e-r diagram of the entities, relationships, attributes into a database supported by the data structure of process, to achieve entity integrity and referential integrity and user-defined integrity. Involving the main business of design data table: the administrator information table, the customer information table, logistics vehicles information table, invoice information table, etc.

The logistics distribution system run the simulation , for suppliers search supply of goods which conform to the owner transport line, for truck drivers search options which are in accordance with the shipper delivery destination, as shown in Figure III.

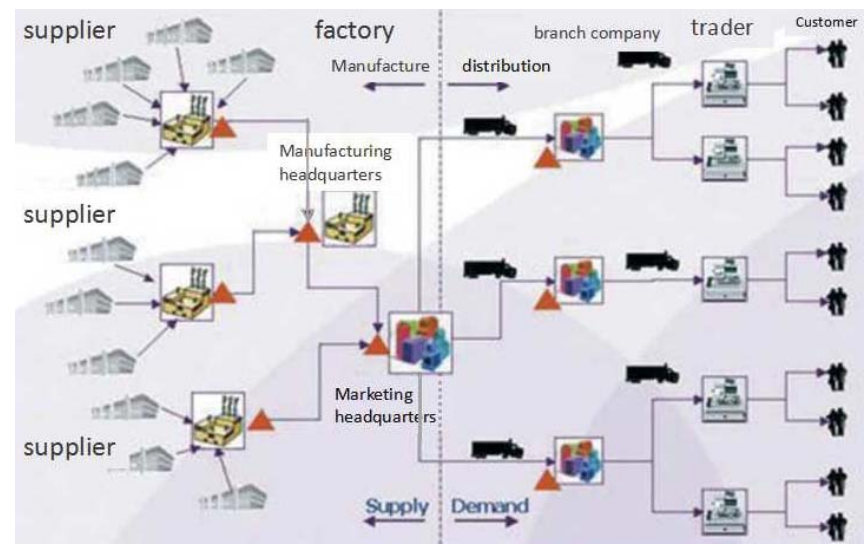

FIGURE III. SIMULATION RUN FIGURE 
Taking Dalian City as a model, Dalian city is divided into multiple zones, each zone has main road. when the system is running, position is provided for supplier and truck drivers as a criteria, let the spaced closer supplier and truck drivers random matching, and open the map mode, make the whole matching and the receiving process more efficient, more convenient. As shown in Figure IV.

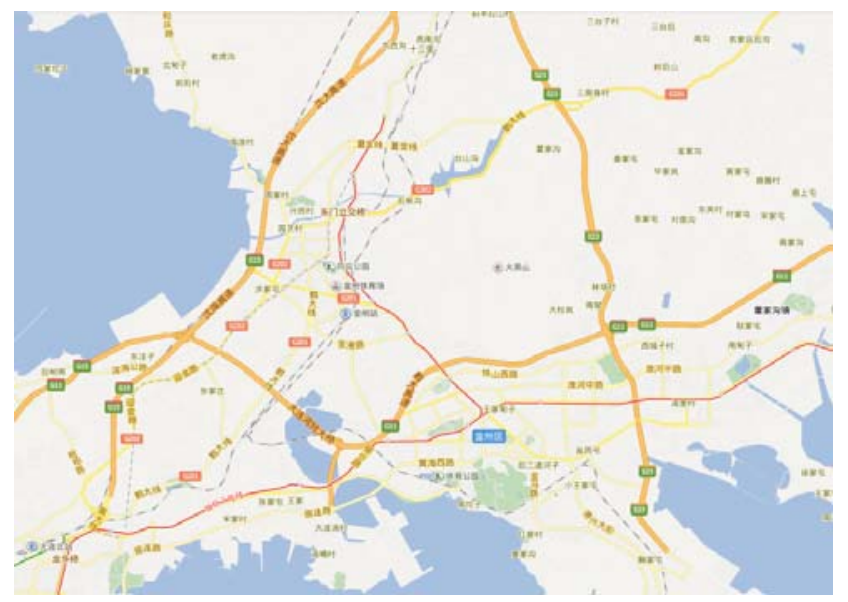

FIGURE IV. GENERAL SITUATION IN DALIAN

System in the design and development, giving full consideration to the user's specific conditions and the use of the operation, not only is possible in theory, more important is available in practice, better adapting to the needs of users .At the same time, reducing the failure rate to the lowest , ensuring that the system is reliable, the system has high MBTF ( Mean Time Between Failure) and low MTBR (Mean Time Between Rate), the system provides fault tolerance design, fault detection and recovery method, when in the network, a hardware or system failure, providing different levels of disaster service. System involves a variety of data related to the interests of the various departments and normal operation of the system. System platform through strict process and access control, audit strictly and allocation system permissions, it is forbidden to unauthorized users to access and manipulate. At the same time, as a result of the system running environment is distributed, we will take effective and strict software protection (anti-virus oftware) combined with hardware safety(hardware firewall) to prevent the outside users on the system of attack and destruction.

\section{CONCLUSIONS}

This is an innovative logistics distribution scheme, it has the following advantages: through the position, supply of goods, capacity status, line and so on, using big data to complete real-time information intelligent push technology, through the real-time positioning service, to provide more accurate position for both the owner and the driver, track both parties, safe and efficient, providing the online payment for both parties, increasing trading capital safety, account and withdraw quickly.

\section{ACKNOWLEDGEMENTS}

This work was supported by "National Undergraduate Training Programs for Innovation and Entrepreneurship of Dalian Nationalities University”.

\section{REFERENCES}

[1] Feng Haiyang, He Jialiang, Zhang Lijun, Meng Fangang, An Application of RFID Technology in Traffic Congestion Management[C] Applied Mechanics and Materials, 2014, vol.513-517, pp.3954-3957.

[2] Shen Peng, He Jialiang, Feng Haiyang, A Solution of Vehicle Emission Inspection Using RFID Technology[C]. 2014 International Conference on Vehicle, Mechatronics and Information Technologies II. Applied Mechanics and Materials, 2014, vols.543-547, pp.946- 949.

[3] Wang Huan, He Jialiang, Pan Yu. A Solution Framework in Traffic Congestion Management Using RFID Technology[C]. International Industrial Informatics and Computer Engineering Conference (IIICEC 2015). 2015, pp.1098-1101. Atlantis Press.

[4] He Jialiang, Shen Peng, Xu Zhiqiang. Inspection Solution of Unlawfully Modified Vehicle by Sensor-RFID Technology[J]. Sensors and Transducers Journal. 2013, vol.25, pp.197-202.

[5] He Jialiang, Xu Zhiqiang. Authentication and Search Mechanism for Diffusing RFID-Sensor Networks[J]. International Journal of Sensor Networks. 2013, vol.14, no.4, pp. 211-217. 